Check for updates

Cite this: RSC Adv., 2019, 9, 9838

\title{
An anisotropic three-dimensional electrospun micro/nanofibrous hybrid PLA/PCL scaffold
}

\author{
Xufeng Dong, (D) *a Jingying Zhang, ${ }^{\mathrm{b}}$ Lu Pang, ${ }^{\mathrm{a}}$ Junting Chen, ${ }^{\mathrm{b}}$ Min Qi, ${ }^{\text {a }}$ Shijie $\mathrm{You}^{\mathrm{c}}$ \\ and Nanqi Ren ${ }^{c}$
}

Although the electrospinning method has been developed to prepare nanofibrous scaffolds, their isotropic structure, low porosity and small pore size prevents them from wide application, especially for anisotropic tissues. In this study, a modified electrospinning receiving system with a rotating mandrel and a water bath is developed. Compared with the nanofibrous scaffold prepared by the common electrospinning system, the micro/nanofibrous polylactide/polycaprolactone (PLA/PCL) hybrid scaffold obtained with the modified system presents anisotropic structure, promotes porosity and enlarged pore size. The hybrid scaffold consists of oriented microfibers and random nanofibers. SEM images demonstrate its anisotropic 3D structure. Tensile testing results confirm that the hybrid scaffold has anisotropic mechanical properties. Compared with the nanofibrous scaffold, human osteoblast-like MG-63 cells protrude more on the surface of the hybrid scaffold. Actin fluorescence staining confirms that the cells form more actin filaments inside the hybrid scaffold. HE staining indicates that more cells enter the interior of the micro/nanofibrous hybrid scaffold. The CCK- 8 activity test shows an enhanced proliferation activity of cells on the surface of the hybrid scaffold. In conclusion, the novel micro/nanofibrous hybrid scaffold has an anisotropic structure and better biocompatibility than common nanofibrous scaffolds, indicating a promising future for use in anisotropic tissue engineering.

Received 31st January 2019 Accepted 22nd March 2019

DOI: $10.1039 / c 9 r a 00846 b$

rsc.li/rsc-advances
There are some other shortcomings prevent the common electrospun scaffolds with nanofibers from practical applications. During the fabrication process, electrostatic interaction exists among nanofibers, which is harmful to produce scaffolds with enough thickness. ${ }^{8-11}$ As a result, the obtained materials are more like two-dimensional thin films rather than three-dimensional scaffolds. They are suitable for skin repair but difficult to repair the tissues with three-dimensional structures. Besides, the pore size of the common electrospun scaffolds is small $(1-10 \mu \mathrm{m})$, which block the normal cell, whose average size is $10-100 \mu \mathrm{m}$, from infiltrating. ${ }^{12-15}$ Furthermore, smaller pore size and lower porosity would hinder the transporting of nutriment and the excreting of metabolite. ${ }^{16-19}$ To overcome those shortcomings, several methods have been proposed ${ }^{20-22}$ For example, Lee et al. promoted the pore size of MMT/PLLA nanocomposite scaffolds by adding salt particles, but it was difficult to separate out all the salt particles, which would decline their mechanical properties. ${ }^{23}$ Whited et al. developed an apatite-coated electrospun PLLA scaffold with large pore size and high porosity by utilizing a threestep water-soluble PEO fiber inclusion, dissolution, and mineralization process, but it was complex for applications. ${ }^{24}$

To develop an anisotropic scaffold with large pore size and high porosity, in this study, we developed a simple electrospinning receiving system with a rotating mandrel and a water bath. Both polylactide (PLA) and polycaprolactone (PCL) are
116024, China. E-mail: dongxf@dlut.edu.cn

${ }^{b}$ Medical College of Dalian University, Dalian, 116024, China

${ }^{c}$ State Key Laboratory of Urban Water Resource and Environment, Harbin Institute of Technology, Harbin, 150090, China 
biomedical polymers that have been approved by U.S. Food and Drug Administration (FDA). ${ }^{25}$ PLA has a degradation half-life of 6-8 weeks and poor mechanical properties; PCL has a degradation half-life of $\sim 1$ year, and good mechanical and rheological properties due to its special backbone structure. ${ }^{26}$ Therefore, polymer blend with PLA and PCL (PLA/PCL) was used to prepare the scaffolds. The obtained electrospun PLA/PCL scaffolds were composed by oriented micro-fiber bundles and random nanofibers, and presented anisotropic three-dimensional structure that mimics anisotropic tissues. The mechanical properties and biocompatibility of those scaffolds were evaluated and compared with the isotropic PLA/PCL scaffolds obtained via common electrospinning method.

\section{Experimental}

\subsection{Scaffold fabrication}

Polylactide (PLA, $M_{\mathrm{w}}$ : 130 000) was provided by Ningbo Global Biomaterials Co. Ltd. (Ningbo, China). Polycaprolactone (PCL, $\left.M_{\mathrm{w}}: 80000\right)$ was supplied by Shenzhen Esun Industrial Co. Ltd. (Shenzhen, China). N,N-Dimethylformamide (DMF) and dichloromethane (DCM) were purchased from Fuyu Fine Chemical Co. Ltd. (Tianjin, China). All the materials were used without further purification.

The electrospinning precursor solution was prepared by dissolving the PLA/PCL blend (mPLA : $\mathrm{MPCL}=1: 1$ ) within the DCM/DMF mixed solution (VDCM : VDMF $=7: 3$ ) at $10 \mathrm{wt} \%$. The precursor solution was sealed. After PLA and PCL were dissolved completely, the solution was uniformed by magnetic stirring for $12 \mathrm{~h}$. Fig. 1 shows the electrospinning system to prepare the micro/nanofibrous hybrid PLA/PCL scaffolds. The receiving device consists of a water bath and a $4 \mathrm{~cm}$-diameter, $15 \mathrm{~cm}$-long rotating mandrel with a piece of aluminum foil attached on its surface. The PLA/PCL blend solution was loaded into a syringe with a 18G-nozzle (inner diameter: $0.84 \mathrm{~mm}$ ) located vertically above the water bath. The horizontal distance and vertical distance between the nozzle and the axis of the mandrel were $6 \mathrm{~cm}$ and $17 \mathrm{~cm}$, respectively. Therefore, the direct distance between the nozzle and the axis of the mandrel of $18 \mathrm{~cm}$. The syringe was supported on a linear guide slider, which drove the syringe reciprocated along the length-direction

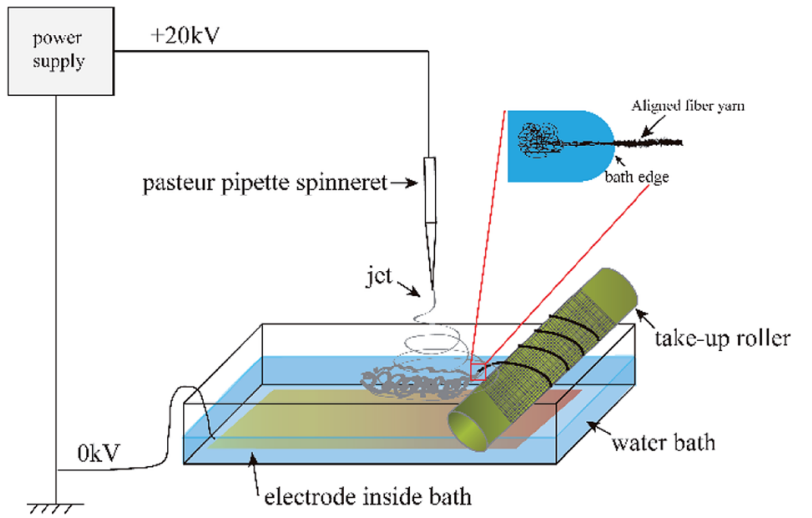

Fig. 1 Schematic diagram of the electrospinning system to prepare the micro/nanofibrous hybrid PLA/PCL scaffolds. of the mandrel at $50 \mathrm{~cm} \min ^{-1}$ during the electrospinning process. A voltage of $20 \mathrm{kV}$ was applied to the nozzle, and the solution jet impelling rate was $0.8 \mathrm{~mL} \mathrm{~h}^{-1}$. During the electrospinning process, some nanofibers directly deposited randomly on the rotate mandrel, while the other nanofibers deposited on the water surface and were continuously collected by the rotating mandrel to form aligned micro-sized fiber bundles. After $4 \mathrm{~h}$, an anisotropic electrospun PLA/PCL scaffold with $\sim 600 \mu \mathrm{m}$ thickness was obtained, as shown in Fig. 2a. For comparison, nanofibrous PLA/PCL scaffolds were obtained by a common electrospinning system, in which the nanofibers were only collected by the rotating mandrel. The direct distance between the nozzle and the axis of the mandrel was also $18 \mathrm{~cm}$. Similar with the process of preparing micro/nanofibrous hybrid scaffolds, a voltage of $20 \mathrm{kV}$ was applied to the nozzle, and the solution jet impelling rate was $0.8 \mathrm{~mL} \mathrm{~h}^{-1}$. After $4 \mathrm{~h}$, the thickness of the scaffold was only $\sim 200 \mu \mathrm{m}$, which was much thinner than the anisotropic scaffold. It indicated the micro/ nanofibrous hybrid PLA/PCL scaffold was much thicker than the nanofibrous scaffold, and the proposed method is more suitable to produce three-dimensional scaffolds. However, for comparison, the nanofibrous scaffolds should have the same thickness as the micro/nanofibrous hybrid scaffolds. Therefore, to obtain nanofibrous PLA/PCL scaffolds with $\sim 600 \mu \mathrm{m}$ thickness (Fig. 2b), the electrospinning process was extended to $14 \mathrm{~h}$.

\subsection{Morphological characterization}

A field scanning electron microscope (FSEM; SUPARR 55, Carl Zeiss, Germany) was used to observe the surface and crosssection morphologies of the scaffolds. Before scanning, all the samples were coated with a thin layer of gold for $45 \mathrm{~s}$. The average diameter of the fibers was calculated from 50 randomly selected fibers. The software Nano measurer was used to analyze the SEM images for this calculation. The mean pore size

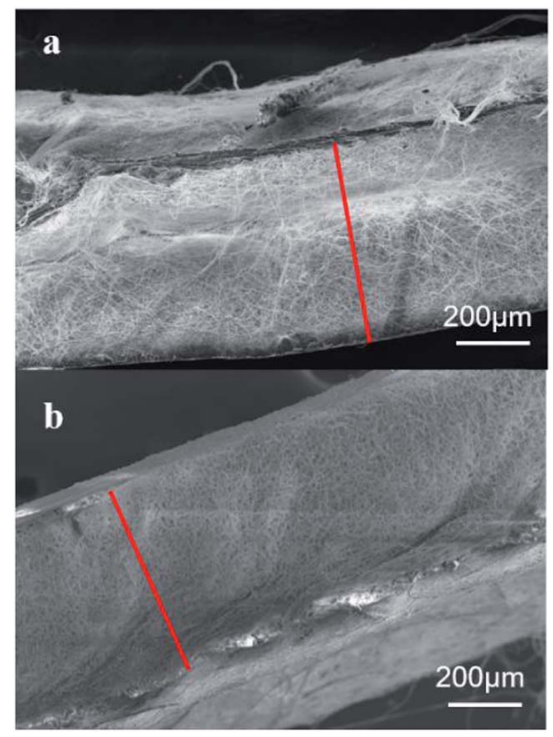

Fig. 2 SEM images of the cross sections of (a) the micro/nanofibrous hybrid scaffold obtained after $4 \mathrm{~h}$ electrospinning and (b) the nanofibrous scaffold obtained after $14 \mathrm{~h}$ electrospinning. 
was obtained by calculating 50 randomly selected pores in the SEM images. The porosity of the scaffolds was measured through a pycnometer method.

Tensile tests. The scaffolds were cut into dumbbell shape with the gauge size of $10 \mathrm{~mm} \times 2 \mathrm{~mm}$ for mechanical properties testing. Tensile tests were performed with an universal material testing machine (WDW3010, Changchun Kexin Testing Machine Co., Ltd.). The tension speed was $10 \mathrm{~mm} \mathrm{~min}^{-1}$.

\subsection{Culturing and seeding of MG-63}

Human osteoblast-like MG-63 cells were purchased from Chinese Academy of Medical Sciences and were as for the cell model. MG-63 was expanded and cultured in basal media with alpha-modified minimum essential medium ( $\alpha$-MEM; Gibco) supplemented with 5\% fetal bovine serum (FBS; Gibco) and 1\% penicillin-streptomycin (HyClone) at $37{ }^{\circ} \mathrm{C} 5 \% \mathrm{CO}_{2}$ until $80 \%$ confluent. Passage 3 cells (P3) were used for this study. Two groups of scaffold samples (3 samples in each group) were cut into $\Phi 14 \mathrm{~mm}$ circle samples. Before cells seeding, the scaffolds were sterilized by UV irradiation for 45 minutes each side. Then the scaffolds were placed in 24-well cell culture plates and incubated overnight with growth media. The MG-63 cells were seeded at a density of $1 \times 10^{4}$ cells per scaffold and cultured at $37{ }^{\circ} \mathrm{C}$ and $5 \% \mathrm{CO}_{2}$ incubator. The growth medium was changed every other day.

\subsection{Cells growths and adhesion}

MG-63 cells were cultured on the nanofibrous scaffolds and micro/nanofibrous hybrid scaffolds (three samples per group) for 1, 2, 3, 5 and 7 days. Scaffolds with cells were coated with gold at a thickness of $5 \mathrm{~nm}$ and examined by the field scanning electron microscopy (FSEM; SUPARR 55, Carl Zeiss, Germany).

\subsection{Fluorescence microscopy and F-actin spread}

Fluorescence images were taken of MG-63 cells seeded on the nanofibrous scaffolds and micro/nanofibrous hybrid scaffolds (three samples per group) after 1, 2 and 3 days post-seeding. The MG-63 cells were stained by Alexa Fluor ${ }^{\circledR} 568$ phalloidin (Thermo Fisher Catalog no. A12380) which is a high-affinity Factin probe. The nucleuses were stained by ProLong ${ }^{\mathrm{TM}}$ Gold Antifade Mountant with DAPI (Thermo Fisher Catalog no. A12380). The fluorescence images were photographed by a confocal laser scanning biological microscope (OLYPUS FV1000, Japan) equipped with a fluorescence filter.

\subsection{Cell proliferation}

Proliferation of MG-63 cells on the nanofibrous scaffolds and micro/nanofibrous hybrid scaffolds for 1, 2, 3 days were determined by the commercially available cell counting kit-8 (CCK-8, Keygentec). MG-63 cells were seeded on the scaffolds (three samples for each group) in 24-well plate for the determined time. Then the cells were detached by trypsin for 5 minutes. The cells suspensions were divided into 4 copies in 96-well plate. The fresh medium containing 10\% CCK- 8 was added and incubated for 4 hours. Then the absorbance was measured at
$490 \mathrm{~nm}$ in a microplate reader (Varioskan Flash, Thermo Fisher, USA).

\subsection{Statistical analysis}

All quantitative values were expressed as means \pm standard deviation (SD). Experiments were performed at least three times and the results of the representative experiments were presented. Statistical analysis was performed by analysis of variance and a $t$-test for independent samples with SPSS version 22.0 software. $P$-values of less than 0.05 were judged to be statistically significant.

\section{Results and discussion}

\subsection{Morphology}

Fig. 3 shows the SEM images of the micro/nanofibrous hybrid PLA/PCL scaffold and the nanofibrous PLA/PCL scaffold. As shown in Fig. 3a and b, the micro/nanofibrous hybrid PLA/PCL scaffold is composed by oriented micro-fiber bundles and random distributed nanofibers, and presents anisotropic structure. The diameters of the micro-fiber bundles were 40.83 $\pm 15.40 \mu \mathrm{m}$, and the diameters of the nanofibers were $871 \pm$ $177 \mathrm{~nm}$. By contrast, the electrospun nanofibrous PLA/PCL scaffold consists of randomly oriented nanofibers, whose diameters were $869 \pm 90 \mathrm{~nm}$, and has isotropic structure, as shown in Fig. 3c and d.

Fig. 4 shows the calculating pore size and porosity of the two kinds of scaffolds. The pore size of the micro/nanofibrous hybrid scaffold (907.67 $\pm 315.64 \mu \mathrm{m}^{2}$ ) is much larger than that of the nanofibrous scaffold $\left(263.42 \pm 89.51 \mu \mathrm{m}^{2}\right)$, as shown in Fig. 4a. Furthermore, the porosity of the anisotropic micro/ nanofibrous hybrid PLA/PCL scaffold (91.89 $\pm 3.48 \%$ ) is higher than that of the isotropic nanofibrous PLA/PCL scaffold (83.4 $\pm 1.79 \%$ ), as shown in Fig. 4 b. It indicates that the micro/ nanofibrous hybrid scaffolds have more promising potential to be applied with respect to the common nanofibrous scaffolds, because larger pore size and higher porosity are beneficial to cell proliferation.

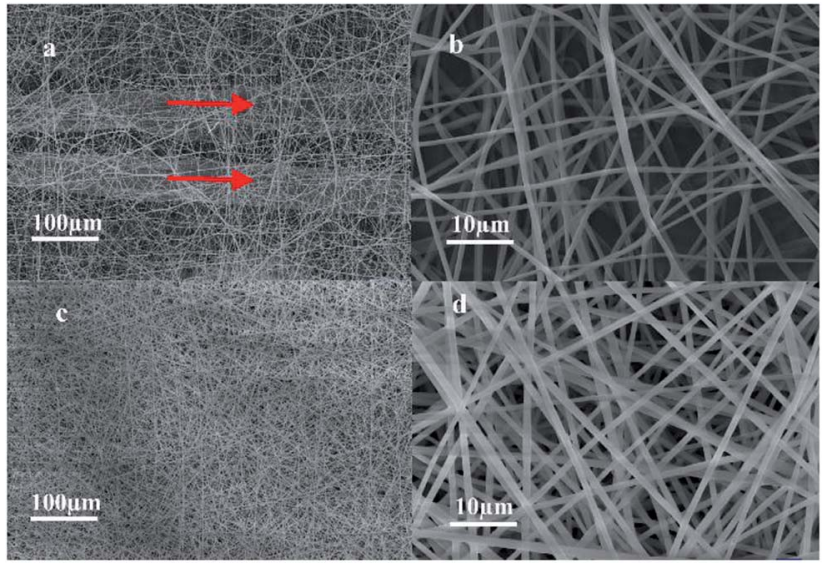

Fig. 3 SEM images of micro/nanofibrous hybrid PLA/PCL scaffold ((a) $500 \times$, (b) $10000 \times$ ) and nanofibrous PLA/PCL scaffold ((c) 500×, (d) $10000 \times)$. 

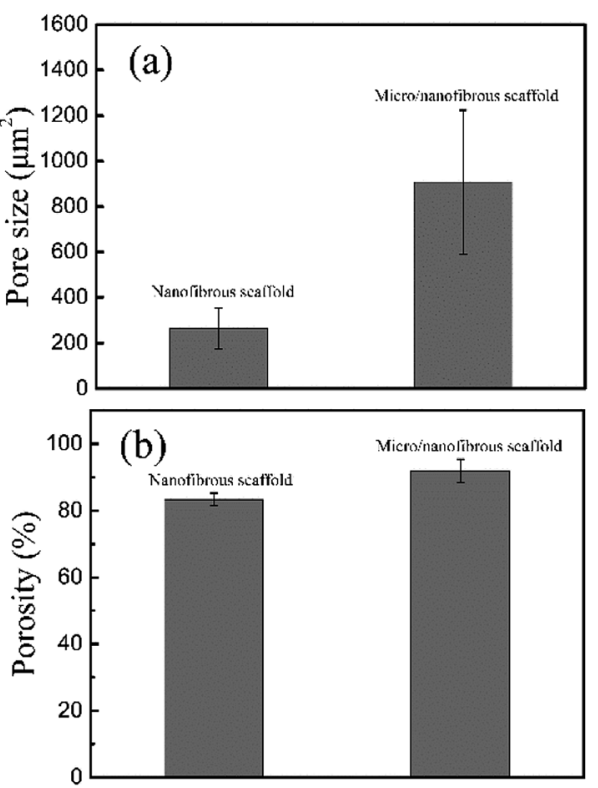

Fig. 4 Pore size (a) and porosity (b) of nanofibrous scaffolds and micro/nanofibrous hybrid scaffolds.

\subsection{Mechanical properties}

Fig. 5 shows the tension stress-strain curves of the micro/ nanofibrous hybrid PLA/PCL scaffold (along the directions parallel and perpendicular to the micro-fiber bundles) and the nanofibrous PLA/PCL scaffold. The obtained mechanical parameters are listed in Table 1. For the micro/nanofibrous hybrid scaffold, the tension strength, elongation at break, and Young's modulus in the direction parallel to the micro fiberbundles are higher than those in the direction perpendicular to the fiber-bundles, indicating anisotropic mechanical properties. Compared with the mechanical parameters along the aligned micro fiber-bundles direction of the micro/nanofibrous hybrid scaffold, the nanofibrous scaffold presents lower tension strength, similar elongation at break, and higher Young's modulus. The anisotropic mechanical properties of the micro/ nanofibrous hybrid scaffolds make them bear different loads in different directions, which indicates they are more suitable

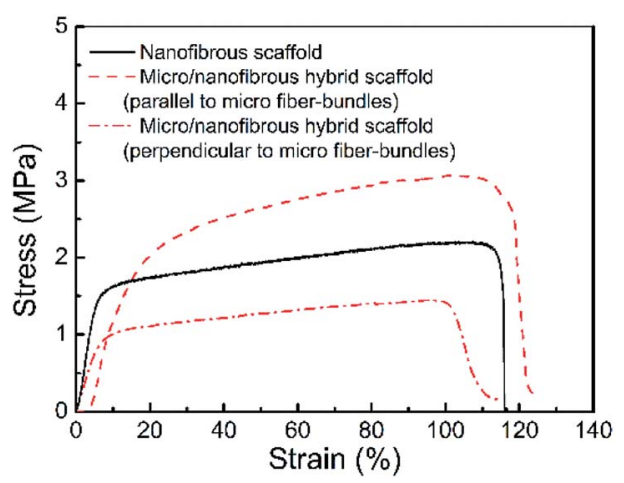

Fig. 5 Tension stress-strain curves of the micro/nanofibrous hybrid PLA/PCL scaffold (along the directions parallel and perpendicular to the micro-fiber bundles) and the nanofibrous PLA/PCL scaffold. for the reparation of anisotropic tissues, such as tendons, muscles, skins and nerves.

\subsection{Cells growth and attachment}

The effect of nanofibrous scaffold and micro/nanofibrous hybrid scaffold on the MG-63 adhesion was viewed by SEM, as shown in Fig. 6. The adhesion and cells growth of MG-63 cells were observed on both of the scaffolds after 1, 2, 3, 5, 7 days of culture. Both scaffolds exhibited excellent bioactivity. In comparison, more extracellular matrix deposits on the micro/ nanofibrous hybrid scaffold (Fig. 6a, c, e, g and i) than nanofibrous scaffold (Fig. 6b, d, f, h and j). Cell filopodia extends along the direction of the scaffold fibers, showing an active growth state. The shape of the cells was like polygonal. Evenly round nuclei can be seen from the images. The surface of the cells secreted a clearly visible extracellular matrix. These figures provided fully evidence that these scaffolds have good

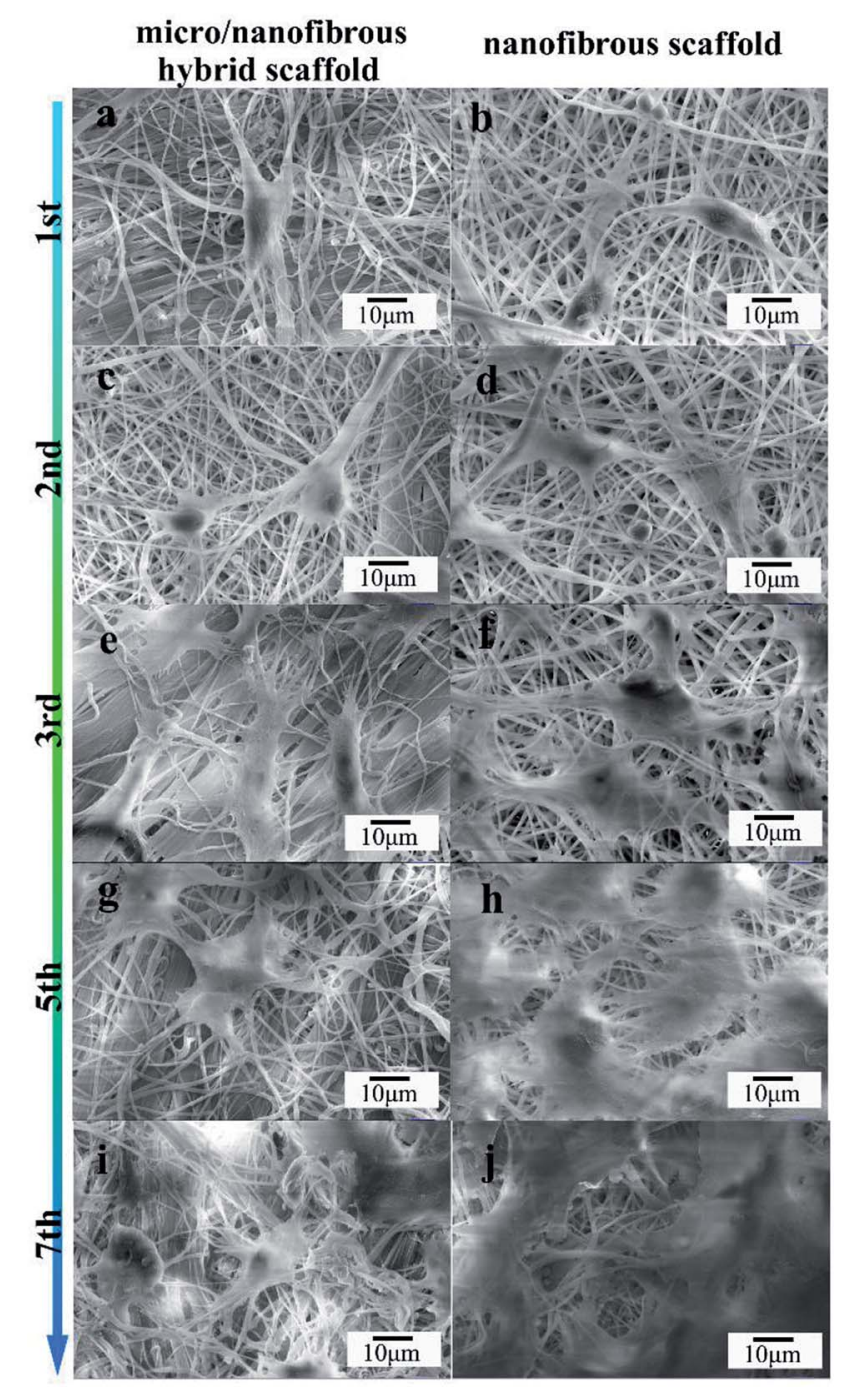

Fig. 6 SEM images of micro/nano fibrous hybrid scaffolds (a, c, e, g and i) and nanofibrous scaffolds (b, $d, f, h$ and j) with MG-63. Showing the microstructure of scaffolds with cells after 1 ( $a$ and b), 2 ( $c$ and d), 3 (e and f), 5 ( $g$ and $h$ ), and 7 ( $i$ and $j$ ) days of culture. 
Table 1 Mechanical properties of nanofibrous and micro/nanofibrous hybrid scaffolds

\begin{tabular}{llrl}
\hline & $\begin{array}{l}\text { Tension strength } \\
\text { (MPa) }\end{array}$ & $\begin{array}{l}\text { Elongation at } \\
\text { break (\%) }\end{array}$ & $\begin{array}{l}\text { Young's } \\
\text { modulus (MPa) }\end{array}$ \\
\hline Nanofibrous scaffold & 2.20 & 103 & \\
Micro/nanofibrous hybrid scaffold (parallel to micro fiber-bundles) & 3.07 & 102 & 295 \\
Micro/nanofibrous hybrid scaffold (perpendicular to micro fiber-bundles) & 1.45 & 96 & 214 \\
\end{tabular}

cytocompatibility and exhibit inducible cellular activity. It can be seen from the 7th day picture that the cells on the surface of the micro/nanofibrous hybrid scaffold are more likely to extend inside the scaffold, but the cells on the surface of the nanofibrous scaffold are linked together almost on the surface. It indicates that the larger pore size of the micro/nanofibrous scaffold is conducive to guide the three-dimensional cell growth.

\subsection{Fluorescence microscopy and F-actin spread}

F-actin of MG-63 was observed to spread rapidly to each of the scaffold surface after 1 day of cell seeding (Fig. 7a and b). F-actin spread well on both of the two kinds of scaffolds, as shown by their change from a rounded shape to a flattened and spread morphology. As the culture time increases, more microfilaments were formed in the cells and the microfilament networks were well-developed. Large fibers can be observed after 3 days cultured. The actin cytoskeletons are also well-organized which indicates the excellent biocompatibility of the scaffolds. It can be seen from Fig. $7 \mathrm{e}$ and $\mathrm{f}$ that on the 3rd day of culturing, the cells on the surface/interior of the scaffolds and the microfilaments in the cells extended in all directions. In comparison, cells on the surface/inside of the micro/nanofibrous hybrid scaffolds had better extension than the nanofibrous scaffolds, which indicates that the anisotropic structure of micro/ nanofibrous hybrid scaffolds can induce microfilament multidirectional growth.

\subsection{Cell proliferation}

The CCK-8 assay was utilized to evaluate the proliferation of MG-63 on nanofibrous scaffolds and micro/nanofibrous hybrid scaffolds for 1, 2, 3 days. The result (as shown in Fig. 8)

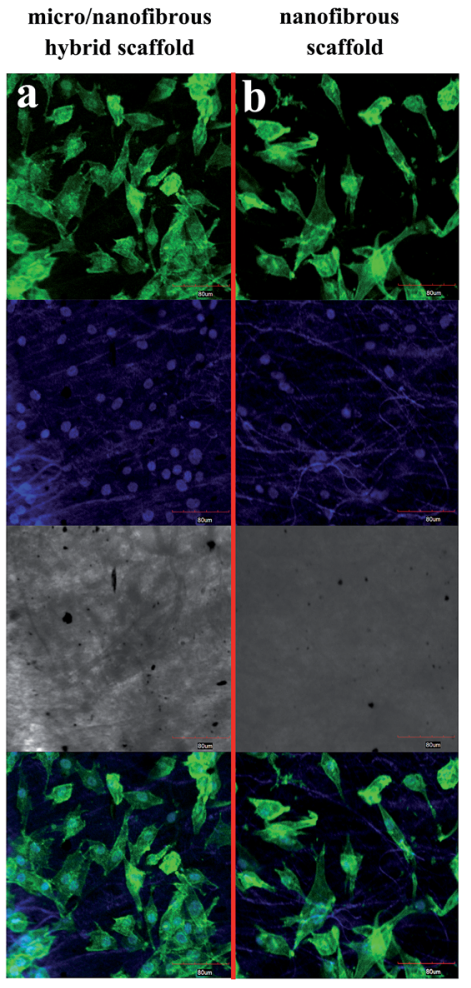

1st day micro/nanofibrous

hybrid scaffold

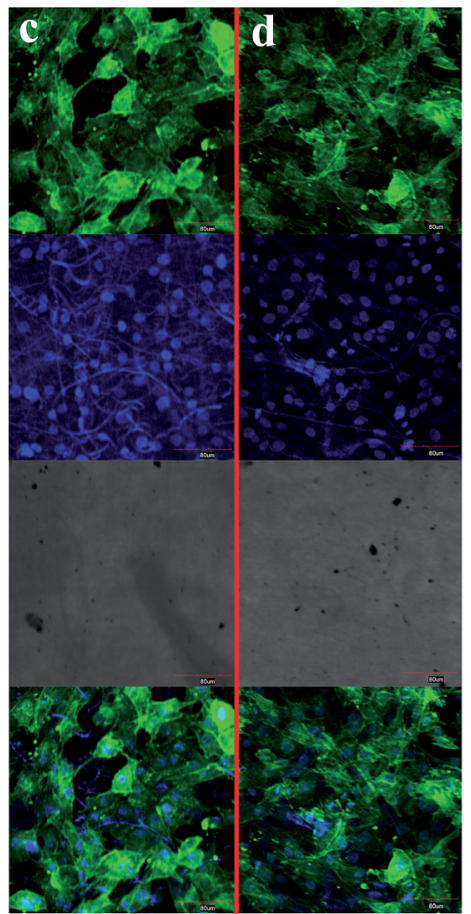

2nd day micro/nanofibrous nanofibrous

hybrid scaffold scaffold

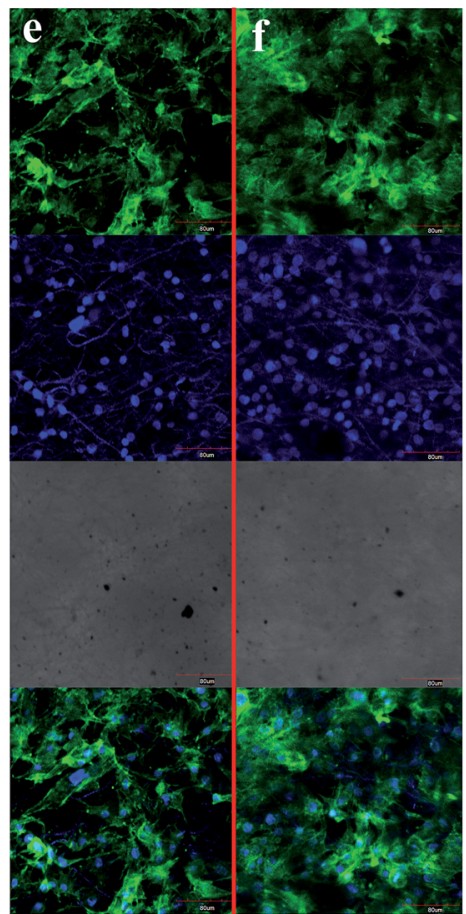

3rd day

Fig. 7 F-actin of MG-63 spread on tree-dimensional (3D) nanofibrous scaffold and micro/nanofibrous hybrid scaffolds. Representative images of viable human MG-63 (labeled with Fluor® 568 phalloidin and DAPI) ((a and b) 1 day after seeding; (c and d) 2 days after seeding; (e and f) 3 days after seeding; ( $a, c$ and e) is micro/nanofibrous composite scaffolds with cells; $(b, d$ and $f)$ is nanofibrous scaffold with cells) Mag $=400 \times$. 


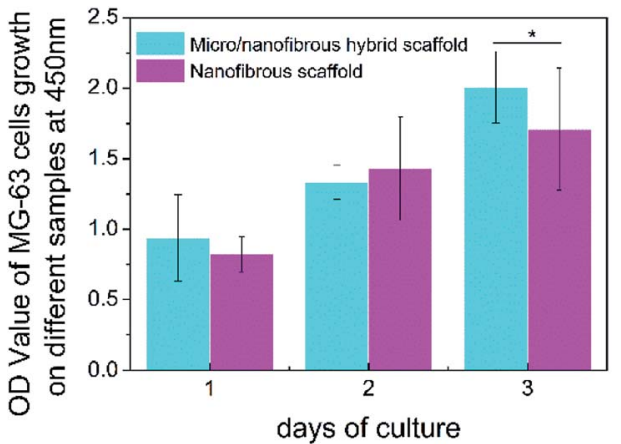

Fig. 8 The proliferation of MG-63 on 3D nanofibrous scaffold and micro/nanofibrous hybrid scaffolds. $(* p<0.05)$.

indicated that there was no significant difference in cell proliferation after the MG-63 cells were cultured on the surface of micro/nanofibrous scaffolds and nanofibrous scaffolds between 1 day and 2 days. From the 1 st day to the 3rd day, the number of cells on both scaffolds continued to increase. On the 3rd day, the proliferative capacity of cells on the surface of the micro/nanofibrous scaffold is significantly better than that of the nanofibrous scaffold $(p<0.05)$. The enhanced proliferative capacity can be attributed to the promoted pore interconnectivity and pore size in the micro/nanofibrous scaffolds.

\subsection{Cell infiltration}

To evaluate the extent of cell migration into the scaffolds, MG63 cells were seeded on the surfaces of micro/nanofibrous hybrid scaffolds and nanofibrous scaffold and cultured for 4 days. HE-stained images of the cross section of the cell-seeded scaffolds are shown in Fig. 9. It indicated the depth of MG-63 cells infiltrated into the inside of the micro/nanofibrous

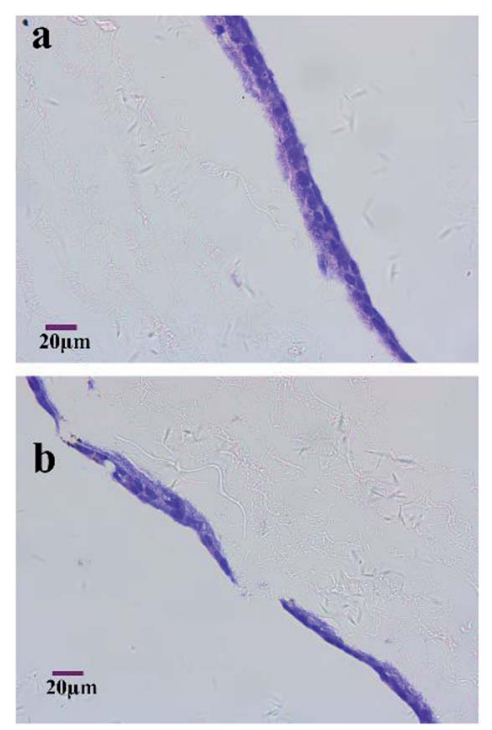

Fig. 9 HE-stained histology images show MG-63 interactions with the micro/nanofibrous hybrid scaffolds (a) and nanofibrous scaffold (b) at 4th day. hybrid scaffold and the nanofibrous scaffold after 4 days' culture. More cells can be clearly seen deep inside in the micro/ nanofibrous hybrid scaffold (Fig. 9a), but there are less cells inside the nanofibrous scaffold comparably (Fig. 9b). The permeability of a scaffold depends on porosity, pore size and pore orientation; when porosity and pore size increases the permeability increases as well. ${ }^{27}$ If the permeability is low the liquid is more likely to travel around rather than through the scaffold and consequently fewer cells enter the scaffold. The porosity and pore size of the anisotropic micro/nanofibrous hybrid scaffolds are higher than that of the isotropic nanofibrous scaffolds, which have shown better permeability for cell infiltration.

\section{Conclusions}

The emerging field of cells based tissue engineering currently offers great promise to meet the clinical need for tissue substitutes. Various criteria have been set out for selection of appropriate 3D scaffolds for tissue engineering application. Achieving surface design and control of biomaterial scaffolds with nanometer- or micrometer-scaled functional films is critical to mimic the unique features of native extracellular matrices, which has significant technological implications for tissue engineering including cell-seeded scaffolds, micro bioreactors, cell assembly, tissue regeneration, etc. By combining a rotating mandrel and a water bath, we developed an electrospinning collecting system to prepare a micro/ nanofibrous hybrid scaffold based on PLA/PCL blend. In comparison with the nanofibrous scaffold obtained by common method, the hybrid scaffold presented anisotropic structure, higher porosity and larger pore size. Mechanical characterization verified the anisotropic structure. Anisotropic structure with hierarchical tri-layered architecture, mechanical anisotropy and biocompatibility are important features in Heart Valve Tissue Engineering (HVTE) ${ }^{28}$ Moreover, anisotropic fiber structure is also a necessary property of electrospun scaffolds for engineering corneal tissues.

Electrospinning, as one of the most effective approaches to fabricate tissue-engineered scaffolds composed of nano- to submicroscale fibers can simulate a native extracellular matrix. In our study, biocompatibility evaluation results indicated that the micro/nanofibrous hybrid scaffolds were more beneficial than nanofibrous scaffolds in attachment, growth, spread, proliferation and infiltration for MG-63 cells. The nanofibers exhibit advantages in improved cells adhesion and proliferation, while microfibers are advantageous in making pore size bigger and promoting cell infiltration. Large pores defined by the microfibers allowed cells to infiltrate the scaffold freely, while the nanofibers facilitated cell spreading and improved cell growth inside the scaffold. The improving cells infiltration of electrospun scaffolds without sacrificing the necessary characteristics are required for tissue repair and regeneration, which ultimately promote their clinical applications. The anisotropic 3D micro/nanofibrous hybrid scaffold has the potential to be used in anisotropic tissues engineering. 


\section{Conflicts of interest}

There are no conflicts to declare.

\section{Acknowledgements}

This research is financial funded by the National Natural Science Foundation of China under the grant number of 51761145031 and 81500890 and Dalian Youth Science and Technology Talents Project under the grant number of 2015R042. Xufeng Dong and Jingying Zhang contributed equally to this work.

\section{Notes and references}

1 E. R. Kenawy, J. M. Layman, J. R. Watkins, G. L. Bowlin, J. A. Matthews, D. G. Simpson and G. E. Wnek, Biomaterials, 2003, 24, 907.

2 S. Agarwal, J. H. Wendorff and A. Greiner, Polymer, 2008, 49, 5603.

3 L. Wang, Y. Wu, B. Guo and P. X. Ma, ACS Nano, 2015, 9, 9167.

4 C. Yang, G. Deng, W. Chen, X. Ye and X. Mo, Colloids Surf., B, 2014, 122, 270.

5 W. E. Teo and S. Ramakrishna, Nanotechnology, 2006, 17, R89.

6 T. J. Sill and H. A. von Recum, Biomaterials, 2008, 29, 1989.

7 T. D. Stocco, N. J. Bassous, S. Zhao, A. E. Granato, T. J. Webster and A. O. Lobo, Nanoscale, 2018, 10, 12228.

8 M. Chen, P. K. Patra, S. B. Warner and S. Bhowmick, Tissue Eng., 2007, 13, 579.

9 N. Bhattarai, D. I. Cha, S. R. Bhattarai, M. S. Khil and H. Y. Kim, J. Polym. Sci., Part B: Polym. Phys., 2003, 41, 1955. 10 Y. Xu, J. Wu, H. Wang, H. Li, N. Di, L. Song, S. Li, D. Li, Y. Xiang and W. Liu, Tissue Eng., Part C, 2013, 19, 925.

11 Q. Yao, J. G. Cosme, T. Xu, J. M. Miszuk, P. H. Picciani, H. Fong and H. Sun, Biomaterials, 2017, 115, 115.
12 G. Zhao, X. Zhang, T. J. Lu and F. Xu, Adv. Funct. Mater., 2015, 25, 5726.

13 K. Garg and G. L. Bowlin, Biomicrofluidics, 2011, 5, 013403. 14 B. A. Blakeney, A. Tambralli, J. M. Anderson, A. Andukuri, D. J. Lim, D. R. Dean and H. W. Jun, Biomaterials, 2011, 32, 1583.

15 S. Jiang, Y. Chen, G. Duan, C. Mei, A. Greiner and S. Agarwal, Polym. Chem., 2018, 9, 2685.

16 B. Sun, Y. Long, H. Zhang, M. Li, J. Duvail, X. Jiang and H. Yin, Prog. Polym. Sci., 2014, 39, 862.

17 A. P. Kishan, A. B. Robbins, S. F. Mohiuddin, M. Jiang, M. R. Moreno and E. M. Cosgriff-Hernandez, Acta Biomater., 2017, 56, 118.

18 G. Wang, D. Yu, A. D. Kelkar and L. Zhang, Prog. Polym. Sci., 2017, 75, 73.

19 J.-O. Jeong, S. I. Jeong, J.-S. Park, H.-J. Gwon, S.-J. Ahn, H. Shin, J. Y. Lee and Y.-M. Lim, RSC Adv., 2017, 7, 8963.

20 Y. Xi, J. Ge, Y. Guo, B. Lei and P. X. Ma, ACS Nano, 2018, 12(11), 10772.

21 W. Gao, W. Jin, Y. Li, L. Wan, C. Wang, C. Lin, X. Chen, B. Lei and C. Mao, J. Mater. Chem. B, 2017, 5(35), 7285.

22 B. Lei, B. Guo, K. J. Rambhia and P. X. Ma, Front. Med., 2018, DOI: 10.1007/s11684-018-0664-6.

23 Y. H. Lee, J. H. Lee, I. G. An, C. Kim, D. S. Lee, Y. K. Lee and J. D. Nam, Biomaterials, 2005, 26, 3165.

24 B. M. Whited, J. R. Whitney, M. C. Hofmann, Y. Xu and M. N. Rylander, Biomaterials, 2011, 32, 2294.

25 Z. Khatri, A. W. Jatoi, F. Ahmed and I. S. Kim, Mater. Lett., 2016, 171, 178.

26 R. T. Zhu, M. H. Tan, P. Zhang, L. Zhang, X. M. Chen and F. W. Yang, Adv. Mater. Res., 2014, 1078, 418.

27 L. Ruiz-Cantu, A. Gleadall, C. Faris, J. Segal, K. Shakesheff and J. Yang, Biofabrication, 2016, 8, 015016.

28 Y. Xue, V. Sant, J. Philippi and S. Sant, Biodegradable and biomimetic elastomeric scaffolds for tissue-engineered heart valves, Acta Biomater., 2017, 48, 2. 